\title{
Retinal oximetry and fractal analysis of capillary maps in sickle cell disease patients and matched healthy volunteers
}

\author{
W. A. J. Birkhoff ${ }^{1,2,3}$ (D) L. van Manen ${ }^{2}$ - J. Dijkstra ${ }^{2}$ - M. L. De Kam ${ }^{1}$ • J. C. van Meurs ${ }^{3,4}$ - A. F. Cohen ${ }^{1,2}$
}

Received: 13 February 2019 /Revised: 19 August 2019 / Accepted: 29 August 2019/Published online: 16 September 2019

(C) The Author(s) 2019, corrected publication 2019

\begin{abstract}
Purpose Fractal analysis can be used to quantitatively analyze the retinal microvasculature and might be a suitable method to quantify retinal capillary changes in sickle cell disease (SCD) patients. Retinal oximetry measurements might function as a proxy for the pathophysiology of cerebrovascular diseases. Moreover, hypoxia has an important role in the pathophysiology of diabetic and other retinopathies. However, little is known about the oximetry around the macula in SCD patients. With this study, we explored the feasibility to perform these quantified measurements in SCD patients.

Methods Retinal microvascular and oximetry measurements were performed in eight SCD patients and eight healthy matched controls. Oximetry pictures and non-invasive capillary perfusion maps (nCPM) were obtained by the retinal function imager. Measurements were conducted twice on two different study days. Measured variables included monofractal dimension (Dbox), relative saturation, deoxygenated hemoglobin (deoxyHb), and oxygenated hemoglobin (oxyHb) concentration.

Results No statistically significant differences in vessel density were found in the different annular zones (large vessels, $p=0.66$; small vessels, $p=0.66$ ) and anatomical quadrants (large vessels, $p=0.74$; small vessels, $p=0.72$ ). Furthermore, no significant between-group differences were found in the other different anatomical quadrants and annular zones around the fovea for relative saturation levels and deoxygenated $\mathrm{Hb}$. However, the oxyHb levels were significantly lower in SCD patients, compared with those in matched controls in the temporal quadrants $(p=0.04 ; p=0.02)$ and the superior nasal quadrant $(p=0.05)$.

Conclusions Our study demonstrated the feasibility of multispectral imaging to measure retinal changes in oxygenation in both SCD patients and matched volunteers. The results suggest that in SCD patients before any structural microvascular changes in the central retina are present, functional abnormalities can be observed with abnormal oximetry measurements.
\end{abstract}

Keywords Sickle cell disease $\cdot$ Retina analysis $\cdot$ Microvessels $\cdot$ Image processing $\cdot$ Computer-assisted

\section{Introduction}

Sickle cell disease (SCD) is the most common inherited blood disorder in Europe and worldwide [1, 2]. Because of increasing immigration rates from Northern and sub-Saharan regions of

W. A. J. Birkhoff and L. van Manen contributed equally to this work.

\section{W. A. J. Birkhoff}

WBirkhoff@CHDR.nl

1 Centre for Human Drug Research, Zernikedreef 8, 2333, CL Leiden, the Netherlands

2 Leiden University Medical Center, Leiden, the Netherlands

3 Oogziekenhuis Rotterdam, Rotterdam, the Netherlands

4 ErasmusMC, Rotterdam, the Netherlands
Africa, SCD should be acknowledged as a major public health issue worldwide [3]. SCD is characterized by structural defects of the hemoglobin molecule, leading to acute or chronic anemia and vaso-occlusive crisis (VOC), which can lead to hospitalization and severe organ damage. Several organs might be affected including the kidneys, lungs, cerebrovascular damage, bones, and the retina [4]. In general, SCD is an inherited disease which may manifest itself already at early childhood. Due to chronicity of the disorder, patients may have intermittent live long symptoms and progressive organ failure due to multiple crises and insufficient oxygen perfusion.

Although SCD mostly manifests peripherally in the retina [5], structural and functional abnormalities in the retinal vasculature around the macula have been observed. [6, 7] Insights in these retinal microvascular changes allows for optimal diagnosis and treatment of SCD patients. The retina is known 
for its high demand for oxygen and might therefore function as a proxy for the pathophysiology of cerebrovascular diseases in general, but also for SCD.

Retinal oximetry is a non-invasive imaging technique wherein fundus photography is combined with the use of multispectral filters to determine oxygen saturation in the retinal blood vessels [8]. As abnormal retinal saturation plays an important role in the pathophysiology of retinopathies in several conditions [9], retinal oximetry imaging has been applied in several patient groups over the past decades [10, 11]. Information about the retinal microvasculature can be obtained through the analysis of non-invasive capillary perfusion maps (nCPM) by means of fractal analysis. Fractal analysis provides a global index that quantifies the geometric complexity of the retinal vascular network, summarizing the entire branching pattern, and may be a more sensitive indicator of early microvascular disease compared with overt retinopathy signs [12]. This method has been applied in patients with Diabetes Mellitus [13], Multiple Sclerosis [14], and high myopia [15].

Until now, knowledge about oximetry in retinal tissue around the macula in SCD patients is scarce. Oximetry images and nCPM can be obtained by the retinal function imager (RFI). Previous studies performed with the RFI were only able to perform a qualitative analysis of the oximetry status of the retina [10], whereas quantitative analysis can be more informative about the retinal status. The current study was the first to quantify retinal oximetry measurements obtained by the RFI in SCD patients and matched controls. With this study, we aimed to qualify new RFI-derived quantitative measures of retinal dysfunction in SCD.

\section{Material and methods}

\section{Study population}

Details about the study population, study design, outcome measures, and assessments were extensively described in the study protocol by Birkhoff et al. [7] In brief, retinal measurements were performed in SCD patients (self-reported) $(n=8)$ aged 18-65 years and healthy controls matched for age, ethnicity, gender, and body mass index ( $n=8$ for capillary maps imaging and $n=9$ for oximetry measurements). SCD patients were recruited through advertisements in local papers or by referral from their treating specialist.

\section{Study design}

This was an observational study conducted at the Centre for Human Drug Research (CHDR) in Leiden, the Netherlands. RFI was conducted twice on two study days separated by 1 week. The study protocol was approved by the ethics committee of Leiden University and performed according to the Dutch law on medical research.

\section{Retinal capillary maps imaging}

Retinal microcirculation was quantified using the RFI 3005 (Optical Imaging, Rehovot, Israel). Measurements were performed as described in the study protocol [7]. In brief, one pupil was dilated using tropicamide. The subject remained seated quietly with the head in a headrest, which allowed for the collection of 10-15 series of eight retinal images in 20 degrees field of view over a period of $25 \mathrm{~min}$. Of the eight images per subject, eight were used for fractal analysis of the nCPM. The images were initially edited and exported using Odian browse software (Optical Imaging, Rehovot, Israel). Subsequently, the best image series for each subject was manually selected based on the image quality (i.e., brightness and sharpness) and consequently analyzed.

\section{Image processing}

Fractal analysis of the nCPMs obtained by the RFI was performed with an automated Matlab (The Mathworks, Inc., Natick, MA, USA) script, which was described elsewhere [12]. In brief, acquired capillary maps were processed to separate microvessels from the original image. Subsequently, box-counting fractal analysis was performed on the processed images. Endpoints were defined as vessel density (Dbox) in different annular and quadrantal zones, which represents the counted small and large vessels at the capillary map. Figure 1 demonstrates the image processing of nCPMs.

\section{Retinal oximetry}

Retinal oximetry maps were acquired with use of the RFI by making series of eight retinal images in a field of view of 35 degrees, using a fast filter wheel with four different wavelengths, of which two oxygen-insensitive ( $548 \mathrm{~nm}$ and $586 \mathrm{~nm}$ ) and two oxygen-sensitive ( $575 \mathrm{~nm}$ and $600 \mathrm{~nm}$ ) wavelengths. The eight images were aligned and were acquired at $55-100 \mathrm{~Hz}$. Each series contained two images of four different wavelengths. Dark reference images, captured by keeping the camera shutter closed, were used to correct for non-uniformity. Due to the lack of a reference standard, only relative oxygenation could be calculated. To normalize the images taken at the oxygen-sensitive wavelengths, we used the images taken at isosbestic points, i.e., a specific wavelength, at which the total absorbance of light of the oxygenated and deoxygenated $\mathrm{Hb}$ is equal (Fig. 2). The images taken at $600 \mathrm{~nm}$ were corrected with use of the images taken at $586 \mathrm{~nm}$, thereby creating an image with deoxygenated $\mathrm{Hb}\left(\right.$ deoxy $\left._{\text {rel }}=I_{600 \mathrm{~nm}} / I_{586 \mathrm{~nm}}\right)$. The images taken at $575 \mathrm{~nm}$ were normalized with use of the images taken at $548 \mathrm{~nm}$, thereby creating an image with oxygenated $\mathrm{Hb}\left(\operatorname{oxy}_{\mathrm{rel}}=I_{575 \mathrm{~nm}} /\right.$ 
Fig. 1 A non-invasive capillary perfusion map (nCPM) (a) processed into an acquired capillary map (b) for boxcounting fractal analysis of vessel density
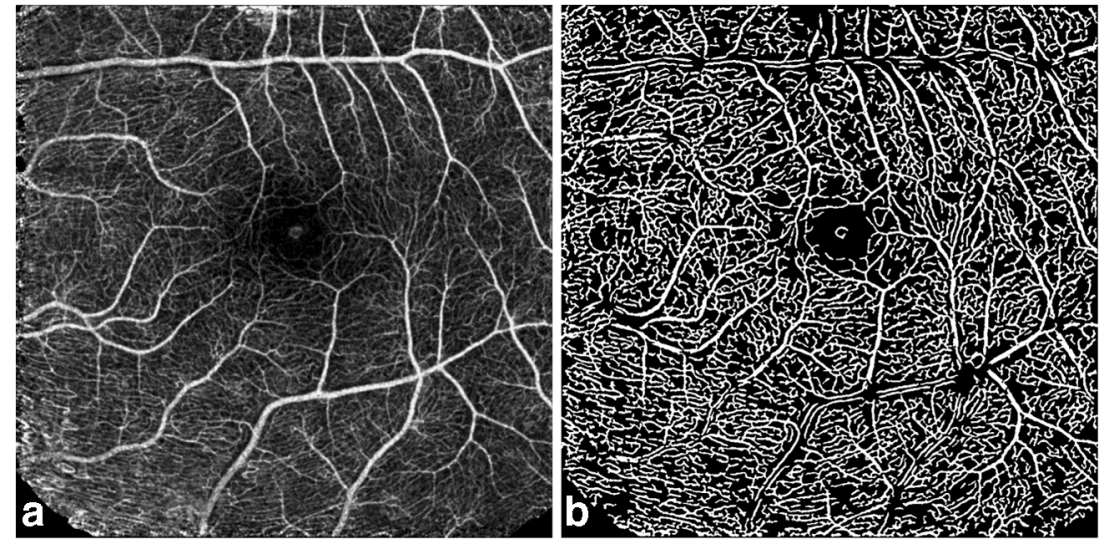

$\left.I_{548 \mathrm{~nm}}\right)$. Consequently, according to the modified Beer-Lambert law, a relative saturation map could be computed $\left(\mathrm{SO}_{2}\right.$ rel $=-$ $\log \left(\mathrm{oxy}_{\text {rel }} / \mathrm{oxy}_{\text {rel }}+\right.$ deoxy $\left._{\text {rel }}\right)$ [16]. This relative saturation map was converted into a color-based map, to make interpretation of saturation easier (blue, low saturation; red, high saturation). For the analysis of the oximetry intensities, the best series were manualy chosen for each subject. We selected several regions of interest (ROIs), which were analyzed with use of MeVisLab, version 3.1 (MeVis Medical Solutions AG and Fraunhofer MEVIS, Germany). Circles were drawn showing four different diameters (10 mm, $25 \mathrm{~mm}, 50 \mathrm{~mm}$, and $75 \mathrm{~mm}$ ) around the macula. Four quadrants (superior nasal, inferior nasal, superior temporal, and inferior temporal) were defined. The side of each square, i.e., the size of the quadrants, was determined by taking the distance from the optic disk to the central part of the fovea. Outcomes indicative for the relative oxygen density were expressed per deoxygenated $\mathrm{Hb}$ and oxygenated $\mathrm{Hb}$ in arbitrary units (AU) and multiplied by 10,000 because the analysis in our software (i.e., calculations based on histograms) required integer values. Saturation was calculated from deoxygenated $\mathrm{Hb}$ and oxygenated $\mathrm{Hb}$ according to the modified Beer-Lambert law (Fig. 2).

\section{Statistical analysis}

For repeatedly assessed endpoints (Dbox) of the capillary maps, contrasts between groups were estimated with a mixed model analysis of variance with fixed factors group (SCD patients and matched healthy subjects), day (day 1 and day 8 ), measurement ( 0 and $2 \mathrm{~h}$ ), group by day, group by measurement and group by day by measurement, and subject, subject by day and subject by time as random factors. By using the same subject ID for the matched subjects, comparisons were pairwise. In both groups, the intra subject variability was calculated for Dbox, from the estimated variabilities within the statistical model.

For oxygenation determination, overall intensities (including minimum, maximum, mean, median, interquartile range (IQR)

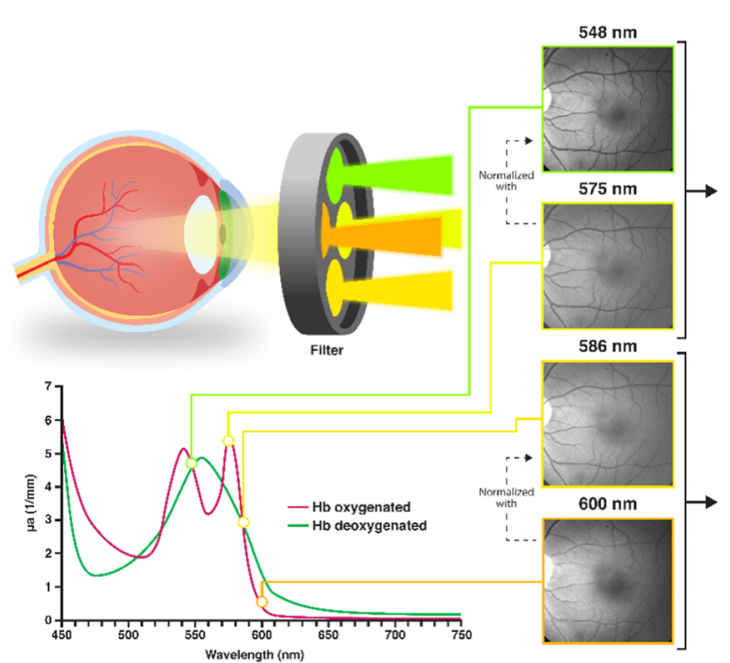

Fig. 2 Schematic overview of oximetry measurements. Schematic overview of oximetry measurements. (1) Images were taken using a fast filter wheel with four different wavelengths, of which two oxygeninsensitive $(548 \mathrm{~nm}$ and $586 \mathrm{~nm})$ and two oxygen-sensitive $(575 \mathrm{~nm}$ and $600 \mathrm{~nm}$ ) wavelengths. (2) The images from oxygen-sensitive

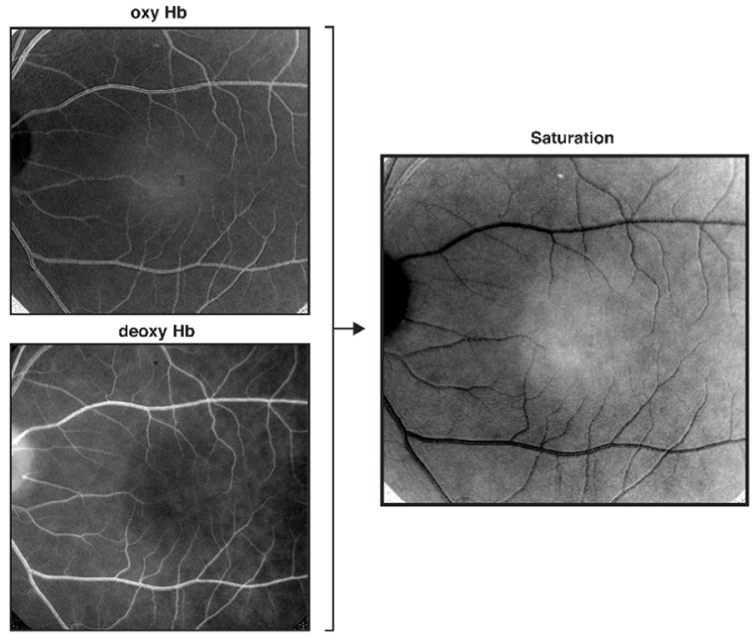

wavelengths were normalized with images from isosbestic points, thereby creating (de)oxygenated images. (3) According to the modified Beer-Lambert law, a relative saturation map was computed, of which different regions where analyzed 
Table 1 Demographics

\begin{tabular}{lll}
\hline & SCD patients $(n=8)$ & Matched controls $(n=8)$ \\
\hline Age (years) & $34 \pm 7.5$ & $31 \pm 7.6$ \\
BMI kg/m & $25.4 \pm 4.8$ & $26.4 \pm 4.2$ \\
Male:female & $0: 8$ & $0: 8$ \\
Systolic blood pressure (mmHg) & $109 \pm 8$ & $116 \pm 18$ \\
Diastolic blood pressure (mmHg) & $65 \pm 6$ & $73 \pm 12$ \\
\hline
\end{tabular}

Demographics obtained during the first occasion for capillary maps measurements. Vital signs were acquired before any measurement had taken place, in both occasions

25-75, peak intensity, and standard deviation) were calculated for each ROI. Each parameter was analyzed using a unpaired Student's $t$ test comparing SCD patients and matched controls; $p$ values $<0.05$ were considered statistically significant. For oxygenation maps, the minimal detectable effect size (MDES) was calculated, assuming a parallel comparison of two groups of eight subjects. All calculations were performed using SAS for windows V9.4 (SAS Institute, Inc., Cary, NC, USA).

\section{Results}

\section{Study population}

Subject characteristics are summarized in Table 1. All included subjects were female.

Fig. 3 Vessel density expressed in Dbox values for all vessels (a), small vessels in different quadrantal zones (b), and annular zones (c) image processing of nCPMs
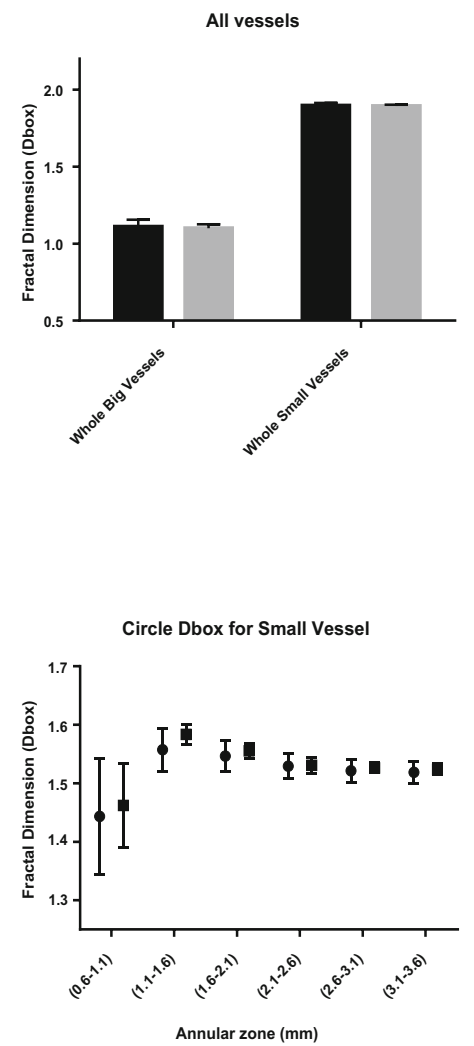

Of all SCD patients, six had homozygous SCD (HbSS) and one heterozygous SCD (HbSE). One subject was uncertain about the SCD subtype. Patients received diverse medicaments, including folic acid, hydroxycarbamide, vitamin D, deferasirox, deferiprone, and acetaminophen. One SCD patient was on hydroxyurea therapy.

\section{Capillary maps}

Figure 3 summarizes the results from the fractal dimension analysis in SCD patients and matched controls, which was performed in the different anatomical quadrants and annular zones around the fovea. We found no significant difference between the SCD patients and the matched controls group with respect to vessel density (Dbox) in large vessels (estimate of the difference, $0.0096 ; 95 \% \mathrm{CI}-0.0376,0.0568 ; p=0.66$ )

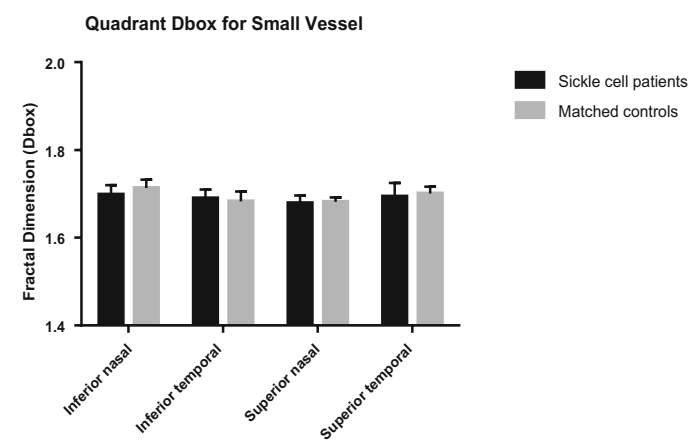

- Sickle cell patients

- Matched controls 
and small vessels (estimate of the difference $0.0033 ; 95 \% \mathrm{CI}-$ $0.0146,0.0212 ; p=0.69$ ) in the different annular zones around the fovea. In addition, we found no significant between-group difference with respect to vessel density in the different anatomical quadrants of large vessels (estimate of the difference $0.0086 ; 95 \% \mathrm{CI}-0.0488,0.0659 ; p=0.74$ ) and small vessels (estimate of the difference $0.0188 ; 95 \% \mathrm{CI}-$ $0.1299,0.0923 ; p=0.72)$.

\section{Oximetry maps}

We found no significant between-group difference in the different anatomical quadrants and annular zones around the fovea with respect to relative saturation levels and deoxygenated $\mathrm{Hb}$. Oxygenated $\mathrm{Hb}$ levels were significantly lower in SCD patients compared with those in matched controls in the temporal quadrant 2 (effect size $(E S)=850.3 ; 95 \%$ CI 69.16 , $1631 ; p=0.04)$, temporal quadrant $3(\mathrm{ES}=919.9 ; 95 \% \mathrm{CI}$ $171.4,1668 ; p=0.02$ ), and the superior nasal quadrant 1 $(\mathrm{ES}=763.8 ; 95 \% \mathrm{CI} 16.03,1512 ; p=0.05)$. Table 2 provides an overview of all retinal oximetry outcomes in different quadrants and annular zones.

\section{Variability and minimal detectable effect size}

Intra subject variability was small for most capillary map measurements, with a coefficient of variation $(\mathrm{CV})<10 \%$. Based upon a parallel comparison of the two groups of eight subjects, the calculated MDES for oximetry measurements in the retina were $173 \mathrm{AU}$ for saturation maps; $198 \mathrm{AU}$ for oxygenated $\mathrm{Hb}$ maps, and $530 \mathrm{AU}$ for deoxygenated $\mathrm{Hb}$ maps.

\section{Discussion}

In this study, we explored the performance of the RFI to study retinal oximetry measurements and to quantify capillary maps in SCD patients and matched healthy controls. A significant difference was observed for oxygenated $\mathrm{Hb}$ maps in the perifoveal quadrants. Oxygenated $\mathrm{Hb}$ levels were significantly lower in SCD patients compared with those in matched controls. We found no significant difference in vessel density, saturation, and deoxygenated $\mathrm{Hb}$ maps between SCD patients and matched controls in all anatomical quadrants and annular zones.

Table 2 Oxygen density measures in different quadrants and annular zones

\begin{tabular}{|c|c|c|c|c|c|}
\hline & & SCD patients $(n=8)$ & Matched controls $(n=9)$ & Effect size controls VS SCD patients & $p$ value \\
\hline \multirow{3}{*}{$\begin{array}{l}\text { Annular zone } 10 \mathrm{~mm} \\
\text { around the fovea }\end{array}$} & Saturation & $2212(210)$ & $2191(330)$ & -20.52 (95\%CI: $-328.5 ; 287.4)$ & 0.89 \\
\hline & Deoxygenated $\mathrm{Hb}$ & $4609(400)$ & $4738(692.4)$ & $128.6(95 \% \mathrm{CI}-503.9 ; 761.2)$ & 0.67 \\
\hline & Oxygenated $\mathrm{Hb}$ & $18,670(958)$ & $19,380(595)$ & $710.1(95 \% \mathrm{CI}-124.0 ; 1544)$ & 0.09 \\
\hline \multirow{3}{*}{$\begin{array}{l}\text { Annular zone } 25 \mathrm{~mm} \\
\text { around the fovea }\end{array}$} & Saturation & $2170(189)$ & $2130(317)$ & $-40.54(95 \%$ CI $-331.6 ; 250.5)$ & 0.77 \\
\hline & Deoxygenated $\mathrm{Hb}$ & $4561(362)$ & 4749 (708) & $188.3(95 \%$ CI $-444.3 ; 820.9)$ & 0.53 \\
\hline & Oxygenated $\mathrm{Hb}$ & $18,872(995)$ & $19,575(606)$ & $702.8(95 \% \mathrm{CI}-157.6 ; 1563)$ & 0.10 \\
\hline \multirow{3}{*}{$\begin{array}{l}\text { Annular zone } 50 \mathrm{~mm} \\
\text { around the fovea }\end{array}$} & Saturation & $2170(153)$ & $2102(292)$ & $-68.14(95 \% \mathrm{CI}-330.0 ; 193.7)$ & 0.59 \\
\hline & Deoxygenated $\mathrm{Hb}$ & $4579(310)$ & 4577 (609) & $-1.937(95 \% \mathrm{CI}-45.4 ; 541.6)$ & 0.99 \\
\hline & Oxygenated $\mathrm{Hb}$ & $18,940(933)$ & $19,605(658)$ & $665.1(95 \% \mathrm{CI}-186.3 ; 1517)$ & 0.12 \\
\hline \multirow{3}{*}{$\begin{array}{l}\text { Annular zone } 75 \mathrm{~mm} \\
\text { around the fovea }\end{array}$} & Saturation & $2213(133)$ & $2127(284)$ & $-89.19(95 \%$ CI $-336.3 ; 164.0)$ & 0.47 \\
\hline & Deoxygenated $\mathrm{Hb}$ & $4638(292)$ & $4610(594)$ & $-27.63(95 \%$ CI $-555.0 ; 499.7)$ & 0.91 \\
\hline & Oxygenated $\mathrm{Hb}$ & $18,773(861)$ & $19,493(668)$ & 719.7 (95\%CI - 98.17; 1538) & 0.08 \\
\hline \multirow[t]{3}{*}{ Quadrant 1: nasal superior } & Saturation & $2381(168)$ & $2197(280)$ & -183.1 (95\%CI; 440.5-74.24) & 0.15 \\
\hline & Deoxygenated $\mathrm{Hb}$ & $5031(450)$ & $4791(646)$ & -240.2 (95\%CI; 856.5-376.1) & 0.42 \\
\hline & Oxygenated $\mathrm{Hb}$ & $18,729(693)$ & $19,493(691)$ & 763.8 (95\%CI $16.03 ; 1512)$ & 0.05 \\
\hline \multirow[t]{3}{*}{ Quadrant 2: temporal superior } & Saturation & $2378(179)$ & $2273(265)$ & -104.8 (95\%CI 355.9; 146.2) & 0.39 \\
\hline & Deoxygenated $\mathrm{Hb}$ & $4946(474)$ & $4926(601)$ & $-20.33(95 \% \mathrm{CI}-615.0 ; 574.3)$ & 0.94 \\
\hline & Oxygenated $\mathrm{Hb}$ & $18,464(810)$ & $19,314(650)$ & 850.3 (95\%CI 69.16; 1631) & 0.04 \\
\hline \multirow[t]{3}{*}{ Quadrant 3: temporal inferior } & Saturation & $2317(176)$ & $2243(217)$ & $-74.11(95 \% \mathrm{CI}-290.8 ; 142.6)$ & 0.48 \\
\hline & Deoxygenated $\mathrm{Hb}$ & $4722(327)$ & $4823(417)$ & $101.0(95 \%$ CI - 311.1; 513.2) & 0.61 \\
\hline & Oxygenated $\mathrm{Hb}$ & $18,313(755)$ & $19,233(642)$ & 919.9 (95\%CI 171.4; 1668) & 0.02 \\
\hline \multirow[t]{3}{*}{ Quadrant 4: nasal inferior } & Saturation & $2278(185)$ & $2267(293)$ & $-10.86(95 \% \mathrm{CI}-283.4 ; 261.7)$ & 0.93 \\
\hline & Deoxygenated $\mathrm{Hb}$ & $4720(374)$ & 4825 (610) & $104.5(95 \%$ CI - 459.9; 668.8) & 0.70 \\
\hline & Oxygenated $\mathrm{Hb}$ & $18,517(1242)$ & $18,983(708)$ & $465.9(95 \%$ CI - 586.2; 1518) & 0.36 \\
\hline
\end{tabular}

Data are expressed as the mean (SD); means represent relative oxygen density in arbitrary units (AU); significant $p$ values are in italic 
RFI is a validated methodology that was designed to directly and non-invasively image the capillary network of the retina in great detail. RFI has been able to measure functional microvascular abnormalities in different patient groups, including SCD patients [7]. Furthermore, several studies have demonstrated the validity of fractal analysis of nCPM in different patients groups as multiple sclerosis patients and subjects with high myopia $[15,17]$. Our results, showing a low intra subject variability for most nCPM endpoints, support these findings.

Although previous studies using optical coherence tomography-angiography (OCT-A) in SCD patients found anatomical differences in capillary structure $[6,18]$, we were not able to repeat these results. This might be due to the fact that the sickle cell patients included in this study were relative healthy with no observed sickle cell retinopathy. This is also supported by a previous study which reported a strong correlation between vessel density around the fovea and retinal thickness, thereby confirming that the eyes of the patients included in this study were relatively unaffected by SCD [6].

Also, the limited sample size compared with that in previous studies [6, 18] might be responsible for this observed difference. Although RFI measurements have been claimed to be equal and even more detailed compared with OCT-A [19], the results might also have been distinct due to the use of different methodologies (fractal analysis versus density analysis). Furthermore, the inclusion of more peri-foveal regions and choroidal OCT-A measurements might have also induced more contrast compared with that in our study. Nevertheless, this methodology was able to observe early retinal changes as expressed by differences in oxygenated $\mathrm{Hb}$.

Our measurements did not show a significant difference between the SCD group and the matched controls with respect to saturation measurements. This might be explained by the fact that the measured differences in endogenous contrast are probably too small for accurate detection. However, we were able to observe a significant difference in oxygenated $\mathrm{Hb}$ in the temporal quadrants of sickle cell patients, which is the location where the disease usually primarily manifests [20]. It is unclear why the superior nasal quadrant also shows statistical difference. As expected, findings in the nasal quadrant however showed a smaller difference compared with the findings in the temporal area. The differences in oxygenation levels more peripheral from the macula might indicate early retinal damage. Furthermore, in our analysis, we found a low inter-subject variability, demonstrated by a low SD, which is in turn reflected by a low MDES. This demonstrates that in case of clinically relevant changes, they would have been observed.

The retina is one of the highest energy-demanding tissues in the body, even surpassing the brain for metabolism, which leads to a high oxygen consumption [21]. Although decreased retinal vessel autoregulation has been reported in SCD patients [22], SCD patients may compensate their lower $\mathrm{Hb}$ and subsequently oxygen-carrying capacity by vascular autoregulatory mechanisms, such as the dilation of the vessels. This is demonstrated by higher retinal blood flow, previously measured in SCD patients [7]. Other techniques have also demonstrated an increased microvascular flow in other organs, thought to be a compensatory mechanism for the anemia [23, 24]. The sickle cell patients in this study had an unimpaired microvasculature around the macula, as demonstrated by the nCPM measurements. Therefore, the autoregulatory capacity of the retinal vessels might be sufficient in these patients, which in turn could lead to normal saturation levels when compared with those in controls. This theory is also confirmed in other studies [25]. Interestingly, our study demonstrates that even before microvascular changes are observed, early retinal changes can already be seen by the reduced level of oxygenated hemoglobin. This is in line with findings from other studies in which differences in other organs such as cerebral oximetry have been reported. [26]

This is the first study to quantitatively analyze multispectral pictures obtained by the RFI, whereas previous studies had only qualitatively analyzed multispectral pictures [10]. In our current analysis, we did not compensate for differences in path length, i.e., absorption and scattering, by the four wavelengths. The expected difference is negligible as the wavelengths are relatively close to each other. In contrast to other research, we measured oximetry as an average in the retinal tissue, not in the individual vessels. This is due to the fact that the current resolution of the RFI does not allow for the reliable quantification in individual vessels using our analysis technology.

A possible limitation of our study is that only females were included; therefore, we cannot exclude gender effects. However, since all SCD patients were matched to control subjects, this does not affect our main conclusions. Although this was an exploratory study, the limited number of participants caused a wider confidence interval for most outcomes. However, due to the low variability and subsequently the low MDES, any clinically relevant contrast would have been picked up. Furthermore, our measurements were performed around the central macula, whereas in SCD, retinal abnormalities are usually primarily observed peri-foveal. In this study, nCPM measurements were performed around the macula, contrary to structural changes that are usually observed in peripheral areas in SCD. We cannot exclude that these patients have abnormalities outside the field of view, more temporal to the fovea. Furthermore, a common drawback of RFI, opposed to OCT-A, is that it only measures the retinal vasculature, not the choroidal vasculature. It should also be noted that the oximetry measurements were still relative, due to the lack of golden standards for retinal oximetry. Nevertheless, our study demonstrated that small peri-foveal abnormalities expressed as subtle changes in oxygenated $\mathrm{Hb}$ can already be picked up by this methodology.

In summary, our study demonstrated the feasibility of multispectral imaging to measure retinal changes in oxygenation in both SCD patients and matched volunteers and that even before a loss of vascular flow can be detected, a reduced level 
of oxygenated hemoglobin might affect retinal function. With the developed methodology, future studies could be performed in which retinal health is monitored by measuring changes in retinal oxygenation in diseases such as Central Retinal Venous Occlusions (CRVO), or retinal detachment.

Acknowledgments We would like to thank Jaspert de Vries for his help in the acquisition of the RFI images.

Funding information LM was funded by the European Union Horizon 2020 Program under grant agreement number 692470 (ASTONISH project) and Topconsortia for Knowledge and Innovation (TKI's) from the Dutch Ministry of Economic Affairs.

\section{Compliance with ethical standards}

Conflict of interest The authors declare that they have no conflict of interest.

Ethical approval All procedures performed in studies involving human participants were in accordance with the ethical standards of the Ethics Committee of Leiden University and performed according to the Dutch law on medical research and with the 1964 Helsinki declaration and its later amendments or comparable ethical standards.

Informed consent Informed consent was obtained from all individual participants included in the study before any measurement took place.

Open Access This article is distributed under the terms of the Creative Commons Attribution 4.0 International License (http:// creativecommons.org/licenses/by/4.0/), which permits unrestricted use, distribution, and reproduction in any medium, provided you give appropriate credit to the original author(s) and the source, provide a link to the Creative Commons license, and indicate if changes were made.

\section{References}

1. Ojodu J, Hulihan MM, Pope SN, Grant AM (2014) Incidence of sickle cell trait—United States, 2010. MMWR Morb Mortal Wkly Rep 63(49):1155-1158

2. Modell B, Darlison M (2008) Global epidemiology of haemoglobin disorders and derived service indicators. Bull World Health Organ $86: 480-487$

3. Weatherall DJ (2010) The inherited diseases of hemoglobin are an emerging global health burden. Blood blood-2010-01-251348

4. Rees DC, Williams TN, Gladwin MT (2010) Sickle-cell disease. Lancet 376(9757):2018-2031

5. Fadugbagbe A, Gurgel R, Mendonça C, Cipolotti R, Dos Santos A, Cuevas L (2010) Ocular manifestations of sickle cell disease. Ann Trop Paediatr 30(1):19-26

6. Han IC, Tadarati M, Pacheco KD, Scott AW (2017) Evaluation of macular vascular abnormalities identified by optical coherence tomography angiography in sickle cell disease. Am J Ophthalmol 177:90-99

7. Birkhoff W, de Vries J, Dent G, Verma A, Kerkhoffs JL, van Meurs AHF et al (2018) Retinal microcirculation imaging in sickle cell disease patients. Microvasc Res 116:1-5

8. Beach J (2014) Pathway to retinal oximetry. Transl Vis Sci Technol $3(5): 2$
9. Stefánsson E, Olafsdottir OB, Einarsdottir AB, Eliasdottir TS, Eysteinsson T, Vehmeijer W et al (2017) Retinal oximetry discovers novel biomarkers in retinal and brain diseases. Invest Ophthalmol Vis Sci 58(6):BIO227-BBIO33

10. Jittpoonkuson T, Garcia PM, Landa G, Rosen RB (2009) Retinal blood-flow velocity and oximetry status monitoring in a central retinal vein occlusion patient. Retin Physician 6(3):50-52

11. Einarsdottir AB, Hardarson SH, Kristjansdottir JV, Bragason DT, Snaedal J, Stefánsson E (2016) Retinal oximetry imaging in Alzheimer's disease. J Alzheimers Dis 49(1):79-83

12. Jiang H, DeBuc DC, Rundek T, Lam BL, Wright CB, Shen $M$ et al (2013) Automated segmentation and fractal analysis of highresolution non-invasive capillary perfusion maps of the human retina. Microvasc Res 89:172-175

13. Zahid S, Dolz-Marco R, Freund KB, Balaratnasingam C, Dansingani K, Gilani F et al (2016) Fractal dimensional analysis of optical coherence tomography angiography in eyes with diabetic retinopathy. Invest Ophthalmol Vis Sci 57(11):4940-4947

14. Jiang H, Delgado S, Tan J, Liu C, Rammohan KW, DeBuc DC et al (2016) Impaired retinal microcirculation in multiple sclerosis. Mult Scler J 22(14):1812-1820

15. Li M, Yang Y, Jiang H, Gregori G, Roisman L, Zheng F et al (2017) Retinal microvascular network and microcirculation assessments in high myopia. Am J Ophthalmol 174:56-67

16. Lu G, Qin X, Wang D, Chen ZG, Fei B editors (2015) Estimation of tissue optical parameters with hyperspectral imaging and spectral unmixing. Medical imaging 2015: biomedical applications in molecular, structural, and functional imaging. International Society for Optics and Photonics

17. Jiang H, Delgado S, Liu C, Rammohan KW, DeBuc DC, Lam BL et al (2016) In vivo characterization of retinal microvascular network in multiple sclerosis. Ophthalmology. 123(2):437

18. Han IC, Tadarati M, Scott AW (2015) Macular vascular abnormalities identified by optical coherence tomographic angiography in patients with sickle cell disease. JAMA Ophthalmol 133(11): $1337-1340$

19. Wang L, Jiang H, Grinvald A, Jayadev C, Wang J (2018) A mini review of clinical and research applications of the retinal function imager. Curr Eye Res 43(3):273-288

20. Stevens TS, Busse B, Lee C-B, Woolf MB, Galinos SO, Goldberg MF (1974) Sickling hemoglobinopathies: macular and perimacular vascular abnormalities. Arch Ophthalmol 92(6):455-463

21. Wong-Riley M (2010) Energy metabolism of the visual system. Eye Brain 2:99

22. van Meurs JC, Schwoerer J, Schwartz B, Mulder PG, Meiselmann HJ, Johnson CS (1992) Retinal vessel autoregulation in sickle cell patients. Graefes Arch Clin Exp Ophthalmol 230(5):442-445

23. Shi PA, Manwani D, Olowokure O, Nandi V (2014) Serial assessment of laser Doppler flow during acute pain crises in sickle cell disease. Blood Cells Mol Dis 53(4):277-282

24. Minniti CP, Delaney KM, Gorbach AM, Xu D, Lee CC, Malik N et al (2014) Vasculopathy, inflammation, and blood flow in leg ulcers of patients with sickle cell anemia. Am J Hematol 89(1):1-6

25. Shahidi M, Felder AE, Tan O, Blair NP, Huang D (2018) Retinal oxygen delivery and metabolism in healthy and sickle cell retinopathy subjects. Invest Ophthalmol Vis Sci 59(5):1905-1909

26. Nahavandi M, Tavakkoli F, Hasan S, Wyche M, Castro O (2004) Cerebral oximetry in patients with sickle cell disease. Eur J Clin Investig 34(2):143-148

Publisher's note Springer Nature remains neutral with regard to jurisdictional claims in published maps and institutional affiliations. 